\title{
Research Status of Vegetation on Flow and Sediment Movement
}

\author{
Guoying $\mathrm{Wu}^{1, \mathrm{a}}$, Jiayi Wang ${ }^{2, \mathrm{~b}}$ and Bingxue Shi, $\mathrm{i}^{3, \mathrm{c}}$ \\ 1 Yellow River Insistute of Hydraulic Research, Zhengzhou 450003, China \\ 2 Yellow River Insistute of Hydraulic Research, Zhengzhou 450003, China \\ 3 School of Water Conservancy and Environmental Engineering, Zhengzhou \\ University,Zhengzhou 450001,China
}

a 284348227@qq.com, b 469512457@qq.com,c1026772054@qq.com

\begin{abstract}
Keywords: flow resistance; turbulence; sediment transport
Abstract: Vegetation in river effect on flow and sediment transport. Based on the main research results, this paper divided the study of effect of vegetation on flow and sediment transport into three aspects: flow resistance, turbulence structure and sediment movement. The flow resistance is mainly affected by the ratio of Manning roughness under different conditions, the turbulence characteristics revealed to Reynolds number in different regions, the problems of sediment transport and suspended is more complicated of vegetation. Research on vegetated flow has made great progress in recent years, but some mechanisms were still not be revealed and the achievements cannot be taken into practice widely. At the end of this article, some limitations and trends were present.
\end{abstract}

\section{Introduction}

It is a common phenomenon that the river containing vegetation, vegetation made the water flow and sediment transport more complex. On the one hand, vegetation contained in the river hindering river flow, causing siltation; on the other hand, it plays a role in adsorbing sediment and protecting riverbank. Vegetation affected the coastal areas and the bottom area's turbulence structure, changed the river bottom's roughness, affected the flow resistance, influenced the law of sediment transport in this area. Compared to the riverbank without vegetation, flow structure is more complex in the riverbank with vegetation, therefore, it is necessary to study the law of flow and sediment transport in the river with vegetation. This article introduce three aspects concluding flow resistance, turbulence structure and sediment transport of vegetation.

Effect of Vegetation on Flow Resistance Characteristics Research .Many experiments show that, for non submerged vegetation, the flow resistance increasing with the increasing of plant density; for submerged vegetation, the canopy resistance increasing with plant density increasing, making water is forced through the area above the canopy flow, causing the water level be higher and canopy velocity be faster. ${ }^{[1 \sim 3]}$ Yan Cheng and Shuyun Qiu ${ }^{[4 \sim 8]}$ through the flume model test, discussed the plant "flexible dam" characteristics, such as arrangement, planting density and the bottom slope effecting on flow, the results show that the plant "flexible dam" can block water flow, intercept sediment, with different plant density and plant length, the effect on blocking water is different. Jie Yan ${ }^{[6]}$ did experiment in order to exploring the effect of flow under the condition that vegetation was in staggered arrangement, 5 different arrangement methods, the results show that the effect of plant "flexible dam" under staggered arrangement blocking water and sand is stronger, with longer dam length and bigger planting density, the area of the "flexible dam" upstream velocity decreasing, the dam height up to the highest, blocking water is more effect.

Cun Wang ${ }^{[9]}$ through simulating flexible vegetation to research the relationship between Manning roughness coefficient $n$ and water depth, put forward the view that with the increasing of water depth, $n$ decreases quickly, and put forward a drag calculation formula regard roughness coefficient $C_{D}$ as a parameter, $C_{D}$ can be calculated by the measured vegetation height, hydraulic radius, water depth and $n$, so that to study its influencing factors. Chow ${ }^{[10]}$ pointed out that only when the water level is lower than a certain water level, vegetation has a significant impact on the flow, thus when water depth is large enough, $n$ tends to be a constant. Can Zhou ${ }^{[11]}$ through establishing stochastic analysis model 
about plant channel roughness, calculated roughness value $n_{e}$ between $0.06 \sim 0.07$, it is corresponded to the empirical value $n_{e}$ between $0.05 \sim 0.08$ in the river with low flow velocity.

Xiaoyan Wang ${ }^{[12]}$ did the flume experiment on flow resistance properties with different plants stiffness, found that for rigid plants, flow resistance is irrelevant to plant flexibility; for the flexible plant, Manning roughness coefficient and drag coefficient decreasing with the increasing of plant flexibility. Kutija and Hong ${ }^{[13]}$ used the most basic one-dimensional hybrid model to study the influencing factors of flexible vegetation on flow resistance, pointed out that because of the bend of vegetation, influence on flexible vegetation resistance is very complexl.

To summarize, there are obvious differences in the characteristics of flow resistance, which are different from the vegetation submergence degree, the stiffness and density.

Effect of Vegetation on Flow Turbulence Characteristics Research. Because of the vegetation existence, the vertical distribution velocity of flow deviates from logarithmic shape, it is complicated with difference vegetation density, stiffness and arrangement methods. In the study of the vertical distribution velocity on flow, the flow is divided into two zones and three zones. Kouwen ${ }^{[14]}$ divided flow into two zones, called plant layer and area that above plant layer. Gourlay, El-Hakim, Carollo and other scholars ${ }^{[15 \sim 7]}$ divided the vertical velocity into three areas, they are the cohesive bottom zone of plant, plant layer and area that above plant layer. The results show that the vertical velocity of the area above plant layer is basically satisfied with logarithmic distribution, and velocity distribution formula of the three regions is not uniform because of the different values of each area.

Zhong Shi, Yanhong $\mathrm{Li}^{[18 \sim 20]}$ studied the turbulence structure at the coastal salt marsh vegetation canopy area, found that flow in vegetation canopy area present triangular structure, called canopy bottom, upper canopy layer, water layer, and canopy area exists reverse velocity gradient, the flow shear stress in the area above the top of the canopy is greater than canopy layer. Kejun Yang ${ }^{[21,22]}$ discussed the overbank flow turbulence structure with channel planting trees, shrubs and weeds. Experiment selected straw plastic, feather and plastic grass to simulate trees, shrubs and weeds, researched the distribution of velocity, turbulent shear stress and turbulence intensity effects of vegetation, found that different vegetation effect on the turbulence intensity dfifferently, with vegetation, turbulence intensity enhanced, it obey "S" shape, "S" shape were in different forms with different vegetation distribution. Cun Wang ${ }^{[23]}$ through flume experiment found that, the flow velocity distribution of emergent plants make its stem and leaves at the inflection point, flow velocity decreases in the upper part, while increased gradually in the lower part; with flow velocity distribution of submerged plants makes plant canopy as the inflection point that presents an inverse "S" shape distribution, velocity increases above the vegetation layer, velocity decreases quickly in the vegetation layer, it is quite obvious for blocking water.

Fusheng $\mathrm{Wu}^{[24]}$ use a specific PVC flexible material to simulate plants, obtained that submerged flexible vegetation vertical velocity distribution does not accord with the exponential distribution; the turbulence flow has obvious anisotropy; flow turbulent exchange is intense in plant canopy junction, the turbulent flow intensity and Reynolds stress reached maximum value. Lianquan Zhao ${ }^{[25]}$ did the flume experiment, found that the bigger the plant density is, the turbulence intensity value become higher and the influence of different kinds of plants tend to be consistent, low plant density relative to small turbulence intensity, but the difference between the vertical distribution is obvious; the bigger plant density is, the turbulent velocity root square fluctuating greatter. Weiwei Song, Jiren Zhou ${ }^{[26]}$ get conclusions that turbulence intensity and the shear stress get maximum at the top of the vegetation in the river, the energy dissipation increases with the vorticity value.

Effect on Sediment Transport of Vegetation. Sediment are normal in the natural river, vegetation effect on sediment transport in river, which made the research more complex.

Hongwu Tang ${ }^{[27]}$ studied the effect on sediment settling velocity of rigid vegetation under hydrostatic condition, the results show that, compared with no plant, when the plant area density greater than 0.071 or the sediment grain size is greater than $0.2 \mathrm{~cm}$, sediment settling velocity were significantly affected, it decreased in different degrees, with sediment particle size and plant density increasing, sedimentation rate decreasing faeter. Zhong Shi ${ }^{[28]}$ measured the mudflat, Scirpus and 
Spartina alterniflora growth environment velocity neared bottom and suspended sediment concentration in the Yangtze River, the results show that the bottom velocity reduced $16 \% \sim 71 \%$ of vegetation in the salt marsh, the suspended sediment concentration is lower than the adjacent mudflat in salt marshes.

Cun Wang ${ }^{[23]}$ through the flume experiment, analyzed the influence on sediment resuspension of emergent and submerged macrophytes, the results show that when the flow intensity is small, both of them can reduce the overlying water turbidity effectively, water turbidity in emergent plants is lower than that in submerged plants, but when the Reynolds number reaches a certain value, the existence of emergent plants aggravated the resuspension of sediments. Fangshe Yang ${ }^{[2]}$ analyze the mechanism of sediment retention through prototype test and flume test. Junning $\mathrm{Li}^{[30]}$ showed that the vertical distribution of suspended sediment concentration decreasing with the increasing plant height, and increasing with the increasing flow. Shengqi Lv ${ }^{[31]}$ studied the influence on bedload movement of submerged flexible vegetation in channel. The results show that when water depth is the same, with the increasing of plant density, sediment incipient velocity decreasing gradually, and a three degree polynomial is correlated well between it and plant density, with the increasing of plant density, bed load transport present a trend that the sand rate increasing first and then decreasing. Supposing the same plant density, the sediment incipient velocity become smaller, and the bedload transport rate become greater with the smaller depth.

\section{Conclusions}

Some achievements have been made by scholars on flow and sediment transport of vegetation, the researches were developed through three kinds of ways, such as laboratory flume experiment, numerical simulation and field measurement mainly, in order to solve problems such as determining Manning roughness coefficient, knowing the turbulent transmission mechanism in plant canopy and the vertical distribution of vertical velocity and sediment concentration. However, owing to the limitation of experimental conditions and the complexity of the actual situation, there is no systematic theoretical or empirical formula until now. The following aspects should be studied deeply: (1) to determine the numerical relationship between the influence factors between Manning roughness coefficient and vegetation height, density through a large number of tests or field measurements, so as to analyze the effect on flow resistance of vegetation. (2) the study of water flow velocity is mainly concentrated in the area above the plant level, and there is little research on the bottom and the bottom of the plant. (3) most of the rivers in the natural with sediment, owing to the complexity of flow movement, it is more difficult to do research on sediment transport of vegetation.

\section{Acknowledgements}

This work was supported by the National Natural Science Foundation of China under Grant (5140090915, 51379086, 5140090916), and Non-profit Industry Financial Program of Ministry of Water Resources (201501003).

\section{References}

[1] Gambi M C, Nowell A R M, Jumars P A: Marine ecology progress series(1990)

[2] Dunn C J, López F, García M H. Hydrosystems Laboratory, Department of Civil Engineering, University of Illinois at Urbana-Champaign(1996)

[3] Zhengbing Chen, Chunbo Jiang. Journal of Tsinghua University(2012), In Chinese.

[4]Yan Cheng, Sen Li, Xiuyun Qiu. Journal of Xinjiang Agricultural University(2003), In Chinese.

[5] Xiuyun Qiu, Yan Cheng, Jie Hou. Water Resources and Hydropower Engineering(2003), In Chinese. 
[6] Jie Yan, Zhu Zhou, Xiuyun Qiu. Journal of Xinjiang Agricultural University (2004), In Chinese.

[7] Feng Liu, Xiuyun Qiu. Journal of Xinjiang Agricultural University (2009), In Chinese.

[8] Feng Liu, Jie Yan, Xiuyun Qiu. Yellow River(2010), In Chinese.

[9] Cun Wang. Hohai University, Nanjing(2003), In Chinese.

[10] Chow, V T. Open Chanel Hydraulic McGraw Hill[M].New York,1959.

[11] Can Zhou, Kun Jiang, Xuyue Hu. China Water Transport (2016), In Chinese.

[12] Xiaoyan Wang. Hohai University, Nanjing (2007), In Chinese.

[13] Kutija V, Hong H T M. Hydraul.Res(1996)

[14] Kouwen N,Unny T E, Hill H M. Journal of the Irrigation and Drainage Division(1969)

[15] Gourlay M R. Journal of tlle Irrigation and Drainage Division(1970)

[16] El-Hakim O, Salama M M. Journal of the Irrigation and Drainage Engineering(1992)

[17] Carollo F G, Ferro V, Termini D. Journal of Hydraulic Engineering(2002)

[18] Zhong Shi. Journal of Sediment Research (1997), In Chinese.

[19] Zhong Shi. The Ocean Engineerig(2001), In Chinese.

[20] Zhong Shi, Yanhong Li. Journal of Shanghai Jiaotong University (2003), In Chinese.

[21] Kejun Yang, Xingnian Liu, Shuyou Cao. Journal of Hydraulic Engineering(2005), In Chinese.

[22] Kejun Yang, Xingnian Liu, Shuyou Cao. Journal of Hydraulic Engineering (2006), In Chinese.

[23] Cun Wang, Chao Wang. Advances in Water Science(2010), In Chinese.

[24] Fusheng Wu. Journal of Hydraulic Engineering (2007), In Chinese.

[25] Lianquan Zhao, Xiaohua Bai, Fengchao Li. Water Resources and Power(2013), In Chinese.

[26] Weiwei Song, Jiren Zhou, Bin Xi. Journal of Water Resources Architectural Engineering (2015), In Chinese.

[27] Hongwu Tang, Shenqi Lv, Jianchuan Long. Journal of Hydraulic Engineering (2007), In Chinese.

[28] Zhong Shi, Shilun Yang, Shen Miao. Journal of Sediment Research(1998), In Chinese.

[29] FangShe Yang, Huaien Li, Lianan Yang. Bulletin of Soil and Water Conservation(2007), In Chinese.

[30] Junning Li, Jurui Yang, Jing Luo. Wetland Science(2012), In Chinese.

[31] Shengqi Lv, Meijiao Guo, Liwei Yuan. Yangtze River(2016), In Chinese. 